\title{
Straining to react: delocalization drives the stability and omniphilicity of [1.1.1]propellane.
}

\author{
Alistair J. Sterling, ${ }^{a}$ Russell C. Smith, ${ }^{b}$ Edward A. Anderson, ${ }^{a *}$ and Fernanda Duarte ${ }^{a *}$ \\ ${ }^{a}$ Chemistry Research Laboratory, University of Oxford, Mansfield Road, OX1 3TA, U.K. \\ banssen P.R.D., 3210 Merryfield Row, San Diego, California 92121, United States
}

KEYWORDS [1.1.1]propellane, strain, omniphilicity, coupled cluster, density functional theory, $\pi$-delocalization

\begin{abstract}
The highly strained caged hydrocarbon [1.1.1]propellane has long fascinated chemists with its seemingly paradoxical stability, yet promiscuous reactivity. This reactivity has been exploited for the synthesis of bicyclo[1.1.1]pentanes (BCPs), motifs that are highly valued in both pharmaceutical and functional material research. However, efficient access to functionalized BCPs from [1.1.1]propellane can be compromized by relatively poor understanding of the underlying mechanisms governing the reactivity of the latter. In this work, a unified model of reactivity is developed that explains the facile addition of nucleophiles, radicals and electrophiles across its bridgehead $\mathrm{C}-\mathrm{C}$ bond. Through Complete Active Space (CAS) calculations, state-of-the-art coupled-cluster methods [DLPNO-CCSD(T)] and DFT approaches, we challenge the hypothesis that reactivity of [1.1.1]propellane is driven by strain relief. Instead, a highly delocalized ground-state electronic structure is proposed, where HOMO-LUMO mixing gives a moldable electron density that results in an omniphilic character. The contrasting nature of the reactivity towards electron-rich and deficient species allows the identification of fundamental guiding principles for the design of novel synthetic pathways and materials from a molecule of timeless appeal.
\end{abstract}




\section{Introduction}

Since Wiberg's first synthesis of [1.1.1]propellane (1, tricyclo[1.1.1.0 $\left.0^{1,3}\right]$ pentane or TCP, Fig. 1a) in 1982, the nature of its $\mathrm{C}_{1}-\mathrm{C}_{3}$ inter-bridgehead bond has been the subject of considerable debate. $^{1-3}$ With three cyclopropane rings fused along an 'inverted' $\mathrm{C}-\mathrm{C}$ axis, this remarkable geometric arrangement nonetheless confers a surprising degree of stability: whereas the homologous [2.1.1] (2) and [2.2.1]propellanes (3) are unstable above $-223{ }^{\circ} \mathrm{C}$ and must be isolated in a $\mathrm{N}_{2}$ matrix, TCP (1) is readily synthesized and can be stored for months in ethereal solution below $0^{\circ} \mathrm{C}$ without appreciable decomposition. ${ }^{4,5}$ Several theoretical and experimental interrogations have sought to explain the ground-state electronic structure of TCP, ${ }^{2,3,6-12}$ most notably the work of Shaik and co-workers who suggested that the stability of $\mathbf{1}$ can be attributed to resonance stabilization between the covalent (repulsive) and ionic (attractive) terms of a $\mathrm{C}_{1}-\mathrm{C}_{3}$ charge-shift bond. ${ }^{13}$ While this model accounts for the observed closed-shell singlet ground state and an unusual positive sign of the Laplacian of the electron density at the $\mathrm{C}_{1}-\mathrm{C}_{3}$ bond critical point, little is understood about the consequences of this unique electronic configuration on reactivity. ${ }^{14}$

In recent years, TCP has become a valuable precursor to bicyclo[1.1.1]pentanes (BCPs), ${ }^{15,16}$ motifs that are attractive bioisosteric surrogates for aryl, ${ }^{17-22}$ alkynyl ${ }^{23}$ and tert-butyl ${ }^{24}$ motifs in medicinal chemistry (Fig. 1b). For example, replacement of a fluorinated arene with a BCP in the Alzheimer's drug candidate Avagacestat (Fig. 1b) enhanced aqueous solubility and cell permeability, while reducing the rate of metabolic degradation and maintaining bioactivity. ${ }^{17}$ Several materials applications have also been developed based around BCPs, including rod-like one-dimensional polymers, ${ }^{25}$ supramolecular spacer units, ${ }^{26}$ liquid crystals ${ }^{27}$ and FRET sensor molecules. ${ }^{28}$ The search for efficient synthetic methodologies to access such materials has led to the develop-

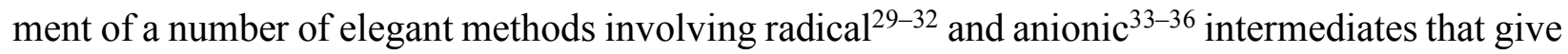
access to a variety of BCP products in a single step from $\mathbf{1}$. To enhance progress in this field, we sought to understand the reactivity of $\mathbf{1}$ from first principles. To date, a handful of mainly experimental studies have employed density functional theory (DFT) to investigate the mechanisms responsible for the addition of radicals and anions to TCP (1). ${ }^{29-31,35,36}$ However, no dedicated theoretical study has tackled the origin of this reactivity. In this work, a unified model of reactivity 
emerges, which bridges the gap between the understanding of TCP (1) as a 'scientific curiosity' to its application in 'real-life' settings. ${ }^{16}$
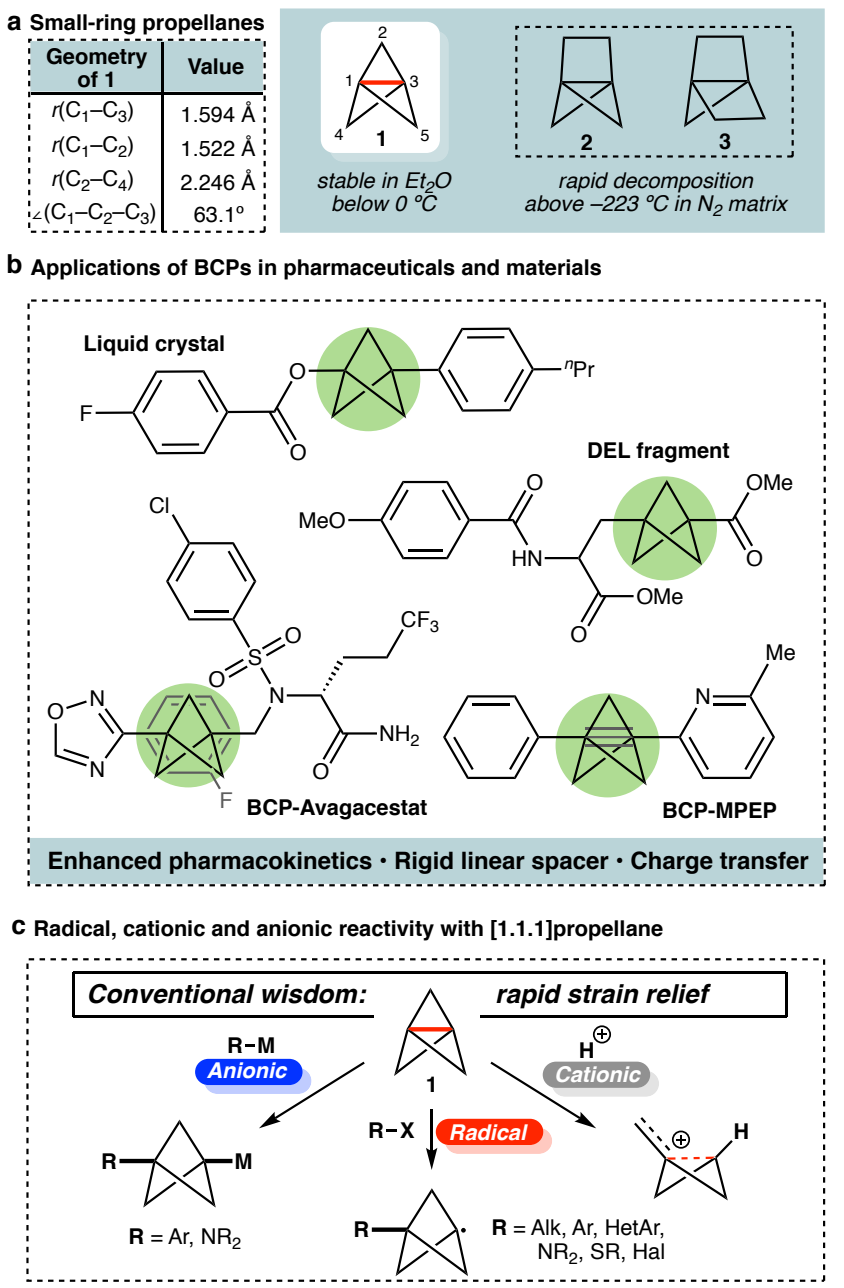

Figure 1. a. [1.1.1]Propellane (1) is stable in ethereal solution below $0{ }^{\circ} \mathrm{C}$ for months, but the corresponding [2.1.1] and [2.2.1]propellanes can only be isolated in an $\mathrm{N}_{2}$ matrix below $-223{ }^{\circ} \mathrm{C}$. Geometric data for 1 were obtained using gas-phase electron diffraction. ${ }^{37} \mathbf{b}$. Applications of $\mathbf{1}$ for the synthesis of bicyclo[1.1.1]pentanes (BCPs). DEL = DNA-encoded library; MPEP = 2-methyl-6-(phenylethynyl)pyridine. c. The omniphilic reactivity of TCP (1) with anions/organometallics (left), radicals (center) and cations (right).

To begin this investigation, we noted that TCP (1) is omniphilic, in that the $\mathrm{C}_{1}-\mathrm{C}_{3}$ bridgehead bond can undergo reactions employing anions, radicals and cations to afford BCPs and exo-methylenecyclobutanes (Fig. 1c). This diverse reactivity has traditionally been ascribed to the high strain energy of $\mathbf{1}\left(\sim 100 \mathrm{kcal} \mathrm{mol}^{-1}\right)$, albeit only $\sim 30 \mathrm{kcal} \mathrm{mol}^{-1}$ of this is predicted to be released 
on cleavage of the $\mathrm{C}_{1}-\mathrm{C}_{3}$ bond. ${ }^{16,38}$ This value is similar to the total strain energy of cyclopropane $\left(\sim 28 \mathrm{kcal} \mathrm{mol}^{-1}\right)$, which displays a distinct reactivity profile. ${ }^{39}$ Moreover, in contrast to standard $\mathrm{S}_{\mathrm{N}} 2$ reactions, the inverted geometry at the site of attack in TCP (1) circumvents the need for planarization. ${ }^{40}$ This observation suggests that the associated energetic cost is likely due to a change in electronics rather than strain relief alone, as is generally believed.

Investigation of these effects in strained systems is not trivial, as even the simple description of the ground state geometry of TCP is challenging for commonly employed Density Functional Theory (DFT). For example, Schreiner et al. have studied the effects of so-called 'inverted' C-C bonding in the propellane family, observing that commonly employed density functionals such as B3LYP and M06-2X fail to describe their ground state geometry. ${ }^{7,41,42}$ Schreiner's work hints that treatment of TCP with DFT may suffer as a result of the unusual electron structure, which arises from correlation effects -the quantum-mechanical interaction of electrons not accounted for by Hartree-Fock (HF) theory. Their work raises the question about the suitability of DFT, which has been extensively used for closed-shell organic molecules, to describe the reactivity of TCP. ${ }^{43}$

Electron correlation effects are commonly divided into dynamic correlation, which describes the spatial interactions between electrons, and static correlation, that comes from the contribution of near-degenerate electronic states that contribute to the overall ground state electronic structure of the molecule. ${ }^{43}$ The DFT exchange-correlation functional $\left(E_{\mathrm{XC}}\right)$ accounts for dynamic correlation effects, but the use of an inexact form of the functional results in the non-physical self-interaction error, where the electron can repel itself. To minimize this error, exact (HF) exchange can be mixed with $E_{\mathrm{XC}}$ (hybrid DFT), and a further perturbative (MP2-like) correction allows a more accurate treatment of non-local correlation (double-hybrid DFT). However, despite tremendous advances in the development of new density functionals, ${ }^{44}$ the treatment of static correlation remains challenging. This is particularly important in systems where substantial mixing of configurations (e.g. small HOMO-LUMO gap) occurs, and standard methods result in spurious geometries and calculated properties. ${ }^{43}$

An alternative approach for the treatment of correlation effects comes from wavefunction theory (WFT). In contrast to DFT, HF theory does not include correlation between electrons with opposite 
spins, and as a result electron density is over-localized. To correct for this error, post-HF methods such as Møller-Plesset perturbation theory and coupled cluster methods include excitations into virtual (unoccupied) orbitals to describe correlation effects. However, these approaches break down if strong correlation plays an important role in the description of the system. A single HF wavefunction cannot adequately describe the electronic structure of the system, and post-HF corrections cannot improve upon this. In these cases, a linear combination of electronic configurations can be used as a reference. In the exact limit, all orbitals are optimized to minimize the electronic energy with respect to all possible electronic configurations (full configuration interaction, FCI). However, due to the exponential scaling of FCI, this method can only be applied to small systems ( $\sim 20$ electrons).

Alternatively, static correlation effects can be accounted for using the complete active space selfconsistent field (CASSCF) method. In CASSCF, the orbital space is divided into three subspaces: an active space within which all possible occupations are considered, an inactive space where all orbitals are doubly occupied and treated self-consistently (i.e. as in HF), and a virtual space where all orbitals are unoccupied. Dynamic correlation is then included by subsequent multi-reference perturbative corrections (e.g. CASPT $2,{ }^{45} \mathrm{NEVPT} 2{ }^{46}$ ) or truncated CI methods (configuration interaction, e.g. MRCI) ${ }^{47}$. This approach still has shortcomings for the treatment of metals, although methods that combine perturbation-corrected CAS and coupled cluster are beginning to offer solutions. $^{48}$

In this work, we investigate the implications of correlation effects on the ground-state properties and reactivity of TCP by comparing HF, DFT, CASSCF and Coupled Cluster [CCSD(T)] methods. To elucidate the influence of strain and electronics on activation barriers, we employ Activation Strain Analysis (ASA) ${ }^{49}$ From this analysis, and contrary to classical expectations, this work demonstrates that TCP reactivity is not driven by strain relief. Instead, it is the change in $\pi$-delocalization over the cage that defines the observed activation profiles. We believe this unified model of reactivity will inspire the design of new synthetic approaches and applications of TCP. 


\section{Computational methods}

All calculations were carried out using the ORCA suite of programs (version 4.1.1). ${ }^{50}$ Groundstate CASSCF optimizations were carried out on 1 with 2 electrons in 2 orbitals, corresponding to the bonding and antibonding combinations of end-on $p$ orbitals between $\mathrm{C}_{1}$ and $\mathrm{C}_{3}$ (Fig. 2a). These results were validated against $\operatorname{CASSCF}(6,6)$ calculations to ensure all static correlation was captured in the smaller active space (see SI §3.1). A relaxed scan of the bridgehead $\mathrm{C}_{1}-\mathrm{C}_{3}$ bond length was carried out using $\operatorname{CASSCF}(2,2)$ with the large def2-QZVPP basis set. ${ }^{51}$ Single-point calculations using both WFT and DFT methods were obtained using these $\operatorname{CASSCF}(2,2)$ geometries to obtain vertical ionization energies and singlet-triplet gaps for a range of $C_{1}-C_{3}$ distances.

Relaxed potential energy surface (PES) scans for the additions of $\mathrm{H}^{+}, \mathrm{CH}_{3}^{+}, \mathrm{H}^{\bullet}, \mathrm{CH}_{3}{ }^{\bullet}$ and $\mathrm{H}^{-}$ were calculated by scanning the forming bond length, $r_{l}$, at the $\mathrm{S}_{0}$ (cationic and anionic) and $\mathrm{D}_{0}$ (radical) $\operatorname{CASSCF}(n, 3) /\left(\right.$ ma-)def2-QZVPP level of theory $\left(n=2\left[\mathrm{CH}_{3}^{+}, \mathrm{H}^{+}\right], n=3\left[\mathrm{CH}_{3}{ }^{\bullet} \mathrm{H}^{\bullet}\right], n=\right.$ $4\left[\mathrm{H}^{-}\right]$, Fig. 2b-d). Subsequent single-point energies with DFT and WFT methods were calculated using the respective $\operatorname{CASSCF}(n, 3)$ geometries $(n=2,3,4)$. The large basis set def2-QZVPP was used for cations and radicals, and the minimally-augmented ma-def2-QZVPP basis set was employed for all calculations involving anionic species.

Given the inherent complexity and computational cost associated with CAS calculations, these systems were benchmarked against DLPNO-CCSD(T) energies for comparison with a range of DFT methods. ${ }^{52}$ No single functional was found to provide optimal results for the whole range of processes studied here. For example, the double-hybrid density functional B2PLYP-D3BJ was found to be optimal for describing radical reactivity (Fig. S4-S5), ${ }^{53-55}$ B2GP-PLYP-D3BJ for anionic reactivity (Fig. S6-S7), ${ }^{56}$ and DSD-BLYP for cationic reactivity (Fig. S8-S9). ${ }^{57}$ Interestingly, the effect of solvation was also found to be vary across these series. While solvation was shown to have a minor effect on the energetics of the radical reaction (Fig. S10), it was found to significantly affect the energetics in cationic and anionic reactions. For this reason, we employed the SMD implicit model, with parameters appropriate for diethyl ether, ${ }^{58}$ for all reactions studied in this work. 
a $(2,2)$ active space for TCP
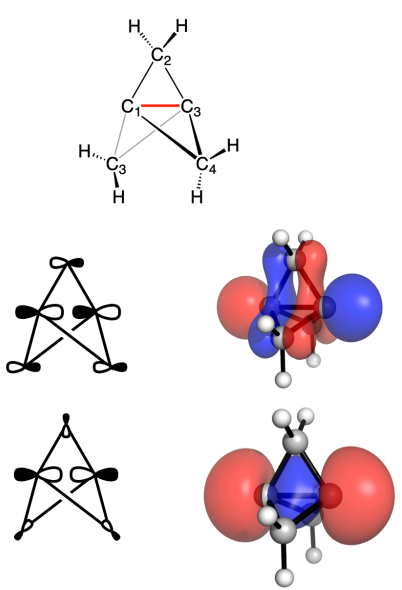

c $(3,3)$ active space for $\mathrm{TCP}+{ }^{\cdot} \mathrm{CH}_{3}$
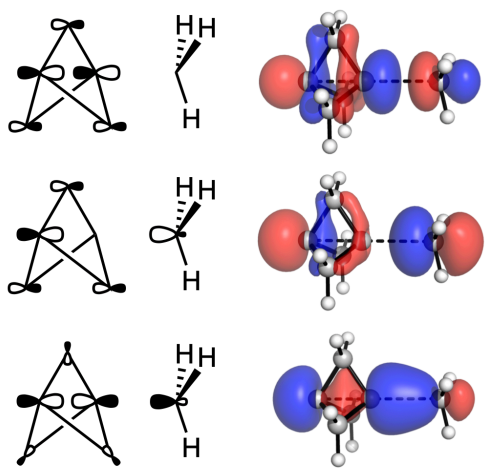

b $(2,3)$ active space for $\mathrm{TCP}+{ }^{+} \mathrm{CH}_{3}$
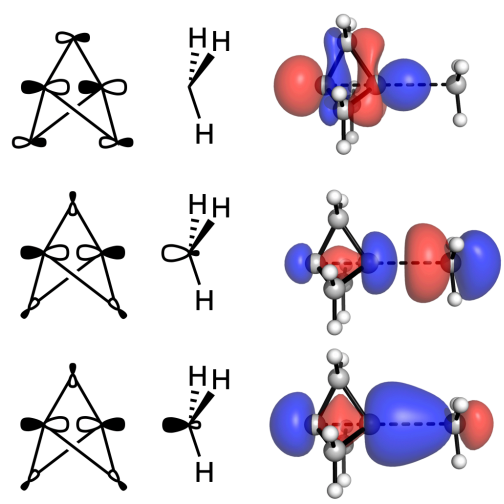

d $(4,3)$ active space for $\mathrm{TCP}+\mathrm{H}^{-}$
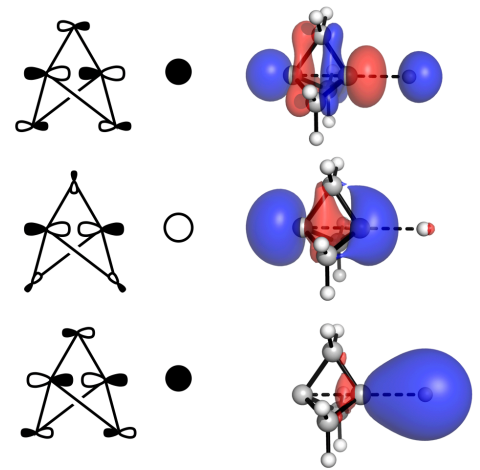

Figure 2. Active spaces used for CAS calculations in this work: a. $(2,2)$ active space used for ground-state electronic structure of TCP (1); b. (2,3) active space for cationic reactivity; c. (3,3) active space for radical reactivity; d. $(4,3)$ active space for anionic reactivity. Orbitals plotted at an isovalue of 0.05 a.u.

As a result of these benchmarking studies, radical reaction free energy profiles were calculated using geometries optimized at the B2PLYP-D3BJ level of theory, and anionic reactivity profiles were calculated with geometries optimized at the B2GP-PLYP-D3BJ level of theory, both using the SMD implicit solvent model. ${ }^{53-56}$ Empirical dispersion corrections are known to be necessary for non-spin component scaled double-hybrid functionals that do not treat long-range correlation well, and benchmarking studies presented in the SI §2.4-2.6 support this finding. ${ }^{57}$ In each of these free energy profiles, the def2-TZVP basis set employed to calculate geometries, ${ }^{51}$ and Gibbs free energy was calculated using Grimme's qRRHO method. ${ }^{59}$ A correction to the Gibbs free energy from $1 \mathrm{~atm}$ to $1 \mathrm{M}$ standard state at $298.15 \mathrm{~K}$ was applied by adding $R \mathrm{~T} \ln (1 / 24.5)=1.89 \mathrm{kcal} \mathrm{mol}^{-1}$ to all solution-phase species. 
Activation Strain Analysis (ASA, also referred to as Distortion/Interaction analysis) ${ }^{40,49}$ was employed to dissect the total energy change $[\Delta \mathrm{E}$ (total)] of each PES up to the transition state into distortion of the geometry of the starting materials $[\Delta \mathrm{E}($ strain)] and electronic interactions $[\Delta \mathrm{E}(\mathrm{int})]$. These electronic interactions include electrostatics, sterics and charge transfer effects. In this work, we divided each reaction system into two fragments (TCP and the approaching species) and calculated the energy of each species as it distorted along the reaction coordinate. Given the definition of $\Delta \mathrm{E}($ total $)=\Delta \mathrm{E}($ strain $)+\Delta \mathrm{E}($ int $)$, we could then analyze the origins of each activation barrier up to the transition state in each case.

\section{Results and Discussion}

\subsection{Characterizing the ground-state electronic structure of [1.1.1]propellane.}

The structure of TCP (1) has been experimentally analyzed by low-temperature X-ray diffraction $^{60}$ and gas-phase electron diffraction, ${ }^{37}$ and from the latter the $\mathrm{C}_{1}-\mathrm{C}_{3}$ bond length was found to be $1.594 \AA$. HF theory underestimates this bond length (1.540 $\AA$, def2-QZVPP basis set), but by including static correlation with $\operatorname{CASSCF}(2,2)$, the cage 'relaxes' to give a length of $1.589 \AA .{ }^{61}$ Inclusion of a doubly excited configuration with CAS permitted the transfer of charge density from the center of the cage onto each bridging carbon atom through $\pi$-delocalization (Fig. 3a), stabilizing the structure by $18.3 \mathrm{kcal} \mathrm{mol}^{-1}$. This effect can be graphically represented using a densitydifference plot (Fig. 3b), where the orange lobe of electron density at the center of the cage indicates an overestimation of electron density with HF. These results suggest that static correlation effects in TCP cannot be captured by a single HF determinant, as the density is not well-described by the HF wavefunction. These results highlight the importance of accurately describing correlation in TCP, and raise the question about the suitability of DFT for describing its reactivity.

We began our investigation by testing whether DFT could accurately reproduce geometric and electronic properties of TCP in isolation. Optimizations using three classes of commonly-employed density functionals (GGA, hybrid GGA and double- hybrid GGA) revealed that BLYP62,63 (GGA) provided an excellent $\mathrm{C}_{1}-\mathrm{C}_{3}$ distance (Fig. 4a, left), while the inclusion of (20\%) HF exchange with B3LYP ${ }^{41}$ underestimated this bond length significantly. Moving to the double-hybrid 
density functional B2PLYP53 appeared to compensate for this error by including virtual orbitals that allowed $\pi$-delocalization to occur, resulting in a decrease in error despite a high proportion of HF exchange in the functional (53\%). These findings explained the excellent geometry from MP2 itself, which was also noted by Schreiner for this system. ${ }^{7}$
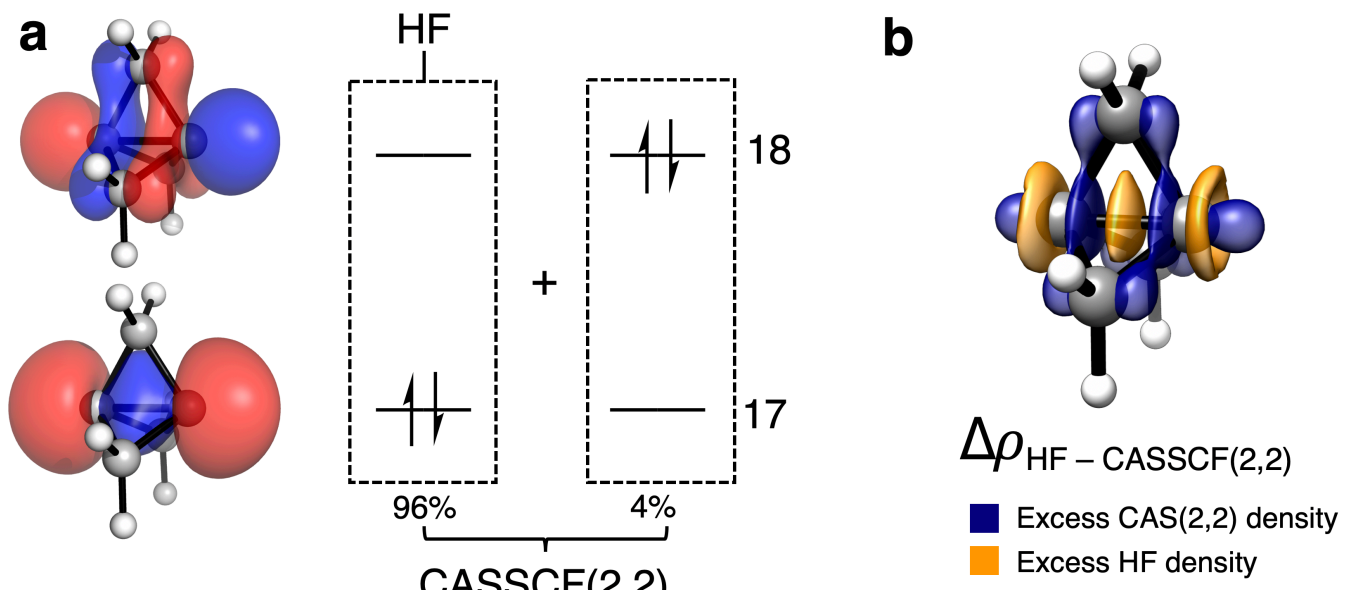

$$
\mathrm{E}_{\mathrm{HF}}-\mathrm{E}_{\mathrm{CASSCF}(2,2)}=\mathbf{1 8 . 3} \mathrm{kcal} \mathrm{mol}^{-1}
$$

Figure 3. a. Important configurations for the ground state of TCP (1) from $\operatorname{CASSCF}(2,2)$ calculations. Orbitals plotted at an isovalue of 0.05 a.u. b. Density difference plot $\left(\Delta \rho=\rho_{\mathrm{HF}}-\rho_{\mathrm{CASSCF}(2,2)}\right)$, plotted at an isovalue of 0.002 a.u. Geometry optimized at CASSCF(2,2)/def2-QZVPP. All calculations use the def2QZVPP basis set.

To test whether these results translated into the electronic properties of 1 , the $\mathrm{S}_{0} \rightarrow \mathrm{T}_{0}$ vertical transition energy was calculated using each method at a range of $\mathrm{C}_{1}-\mathrm{C}_{3}$ distances, to capture longand short-range electronic effects (Fig. 4a, middle). As before, pure DFT (BLYP) performed favorably compared with hybrid DFT (B3LYP); however, inclusion of MP2-like correlation in the functional (B2PLYP) markedly reduced the calculated error. Vertical ionization potentials at each level of theory also yielded similar results. From these three tests, it became clear that $\pi$-delocalization through population of virtual orbitals (i.e. correlation) had a significant impact on the electronic structure of TCP.

Once again, the use of density difference plots was instructive in understanding the origin of these errors (Fig. 4b). Comparison of the BLYP and CASSCF(2,2) electron densities demonstrates that the former underestimates the density along the vertex $\mathrm{C}-\mathrm{C}$ bonds (blue lobes), while it overlocalizes density on $\mathrm{C}_{1}$ and $\mathrm{C}_{3}$. As a result, we anticipated GGA functionals to underestimate the 
strength of the $\mathrm{C}_{1}-\mathrm{C}_{3}$ bond. The inclusion of HF exchange in B3LYP improved the density along the vertex $\mathrm{C}-\mathrm{C}$ bonds; however, it overlocalized density along the $\mathrm{C}_{1}-\mathrm{C}_{3}$ bond (orange lobes), originating from the same effect as in HF itself. Finally, B2PLYP provided an excellent match to the $\operatorname{CASSCF}(2,2)$ density, explaining its improved performance compared with its GGA and hybrid-GGA counterparts.

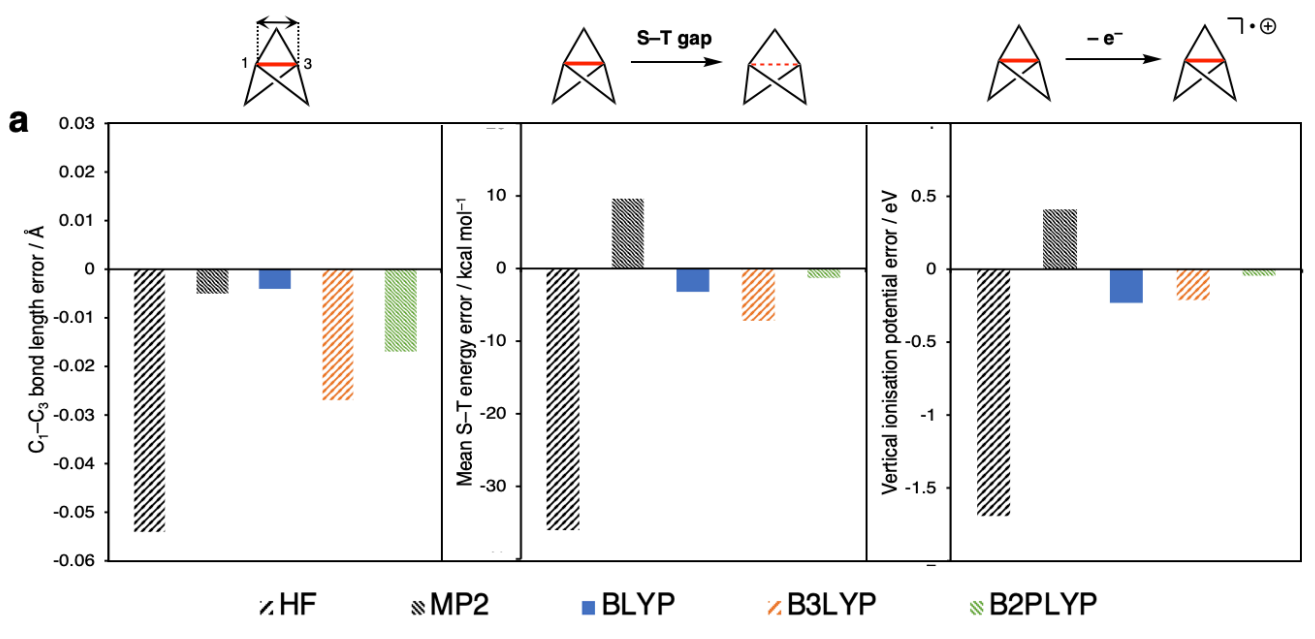

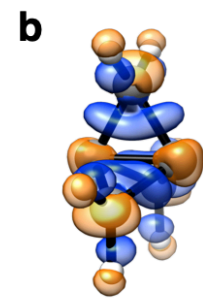

BLYP - CAS

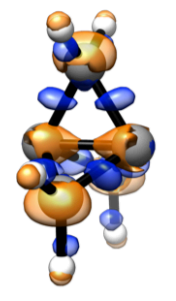

B3LYP - CAS

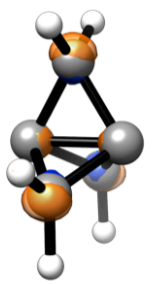

B2PLYP-CAS

$$
\begin{aligned}
& \Delta \rho_{\mathrm{DFT}-\mathrm{CASSCF}(2,2)} \quad \text { Excess CAS(2,2) density } \\
& \text { Excess DFT density }
\end{aligned}
$$

Figure 4. a. Left: Deviation of $\mathrm{C}_{1}-\mathrm{C}_{3}$ bond length of 1 at each level of theory (def2-QZVPP basis set) from the experimental value ${ }^{37}$ of $1.594 \AA$; middle: Average deviation of vertical $\mathrm{S}_{0} \rightarrow \mathrm{T}_{0}$ transitions of 1 at $\mathrm{C}_{1}-\mathrm{C}_{3}$ distances ranging from 1.54 to $2.00 \AA$ at each level of theory, geometries optimized at the CASSCF (2,2)/def2- QZVPP level of theory; right: Vertical ionization potentials (eV) at each level of theory. b. Density difference plots for BLYP, B3LYP and B2PLYP $c$.f. CASSCF $(2,2)$ electron density at an isovalue of 0.01 a.u., all with a def2-QZVPP basis set. 


\subsection{The reactivity of [1.1.1]propellane with anions.}

Having identified a suitable computational methodology, and the beneficial role of $\pi$-delocalization, we next explored the reactivity of TCP. To understand how omniphilicity could emerge from this model we studied a set of established synthetic systems. Reactions of TCP with anions have been reported by Wiberg, ${ }^{64}$ de Meijere, ${ }^{65} \operatorname{Baran}^{33,34}$ and Walsh ${ }^{35,36}$ to access aryl, alkyl and amino BCPs. For example, Baran employed turbo-Hauser amides $\left(\mathrm{NR}_{2} \mathrm{MgCl} \bullet \mathrm{LiCl}, \mathrm{R}=\right.$ alkyl $)$ to form amino-bicyclo[1.1.1]pentanes, where the driving force for the addition was attributed to the relief of strain. However, the surprisingly long reaction times $(16 \mathrm{~h})$ and elevated temperatures (50 ${ }^{\circ} \mathrm{C}$ ) required for the reaction did not fit with the concept of 'rapid' strain relief. Walsh has also studied the anionic reactivity of TCP with dithianes under similar reaction conditions, ${ }^{36}$ complementing synthetic developments with DFT calculations at the M06-2X level of theory. Using this approach, they reported the addition of the anion to TCP to be highly endergonic. These examples caused us to query the importance of strain relief in the reaction of anions with TCP, and to question how a thermodynamically disfavored process could occur in a system thought to be primed to react.

To understand the driving force for the addition of anions to TCP, we analyzed Baran's addition reaction of turbo-Hauser amides with TCP, where fast addition of the amide anion would give a BCP anion that is subsequently quenched upon work-up (Fig. 5a). ${ }^{33}$ For the addition of a model amide anion $\left(\mathrm{NH}_{2}{ }^{-}\right)$to $\mathbf{1}$, we found the formation of the anionic adduct $(\mathbf{B})$ to be reversible $\left(\Delta \mathrm{G}=+1.3 \mathrm{kcal} \mathrm{mol}^{-1}\right.$, Fig. 5b). Only on complexation of this species to a model $\mathrm{MgCl}^{+}$ion did the overall reaction become highly exergonic $\left(\Delta \mathrm{G}=-64.8 \mathrm{kcal} \mathrm{mol}^{-1}\right)$.

The slight endergonicity of the first addition step suggested that, as well as being disfavored by the formation of a less thermodynamically stable anion, the reaction may in fact form a more strained intermediate than TCP itself. We employed Activation Strain Analysis to investigate this hypothesis, and noted that going from 1 to the transition state (TS) $\mathbf{A}$, both $\Delta \mathrm{E}$ (strain) and $\Delta \mathrm{E}$ (int) were positive; in fact $\Delta \mathrm{E}$ (int) was twice the magnitude of $\Delta \mathrm{E}$ (strain) (Fig. 5b). This is highly unusual, as to the best of our knowledge $\Delta \mathrm{E}$ (int) has never been observed to be greater than $\Delta \mathrm{E}$ (strain) for any chemical system. Given that $\Delta \mathrm{E}$ (strain) will only increase past the transition state, we 
concluded that $\Delta \mathrm{E}$ (int) must become more favorable to allow for the formation of a stable product. In fact, analysis of the change in partial charges from $\mathbf{1}$ to TS A indicates that each vertex carbon atom experienced a build-up of charge, suggestive of delocalization effects within the cage (Fig. $5 \mathbf{b})$.

a Proposed mechanism for Baran's strain-relief amination

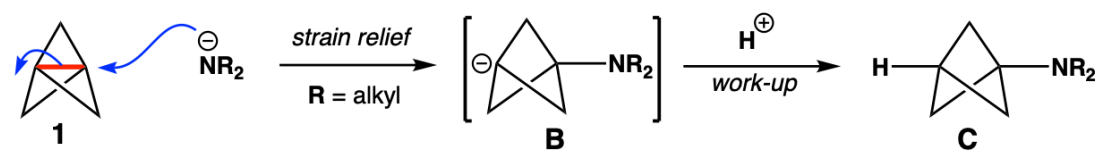

b Revised mechanism: delocalisation over the cage

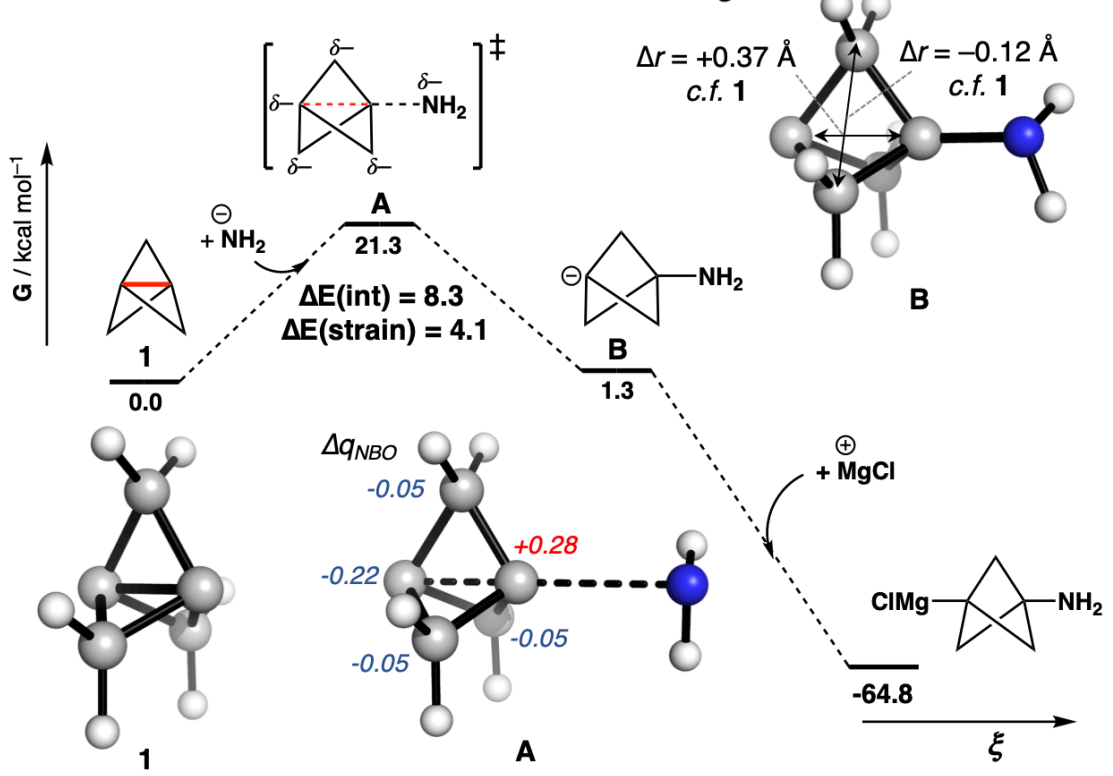

Figure 5. a. Proposed reaction mechanism for Baran's turbo-Hauser amide addition to 1. b. Free energy profile for the addition of $\mathrm{NH}_{2}^{-}$to TCP (1), followed by complexation with $\mathrm{MgCl}^{+}$, calculated at the SMD(THF)-DLPNO-CCSD(T)/ma-def2-QZVPP//SMD(THF)-B2GP-PLYP-D3BJ/ma-def2-TZVP level of theory. $\Delta q_{N B O}(e)$ indicates the change in NBO charge going from $\mathbf{1}$ to $\mathbf{A}$.

Exploring this further, we employed high-level CAS and coupled cluster [DLPNO-CCSD(T)] calculations on a model system to identify how this delocalization manifests over the cage (Fig. 6a). Extending the Activation Strain Analysis along the reaction coordinate, we observed that the increase in energy on approach of the anion to TCP arises from long-range repulsive electronic interactions, which has little effect on the geometry. However, closer to the TS, short-range effects dominate, leading to a sharp increase in the distortion of the cage causing significant electronic stabilization. Analysis of the $\operatorname{CASSCF}(4,3)$ active orbitals along this path showed that the lobe of the $\sigma$-bonding $\mathrm{MO}$ of $\mathbf{1}$ pointing at the nucleophile retreated away from the approaching anion 
(Fig. 6c), distorting to allow $\pi$-delocalization to occur as $p$ orbitals on each vertex carbon atom became involved in the bonding.

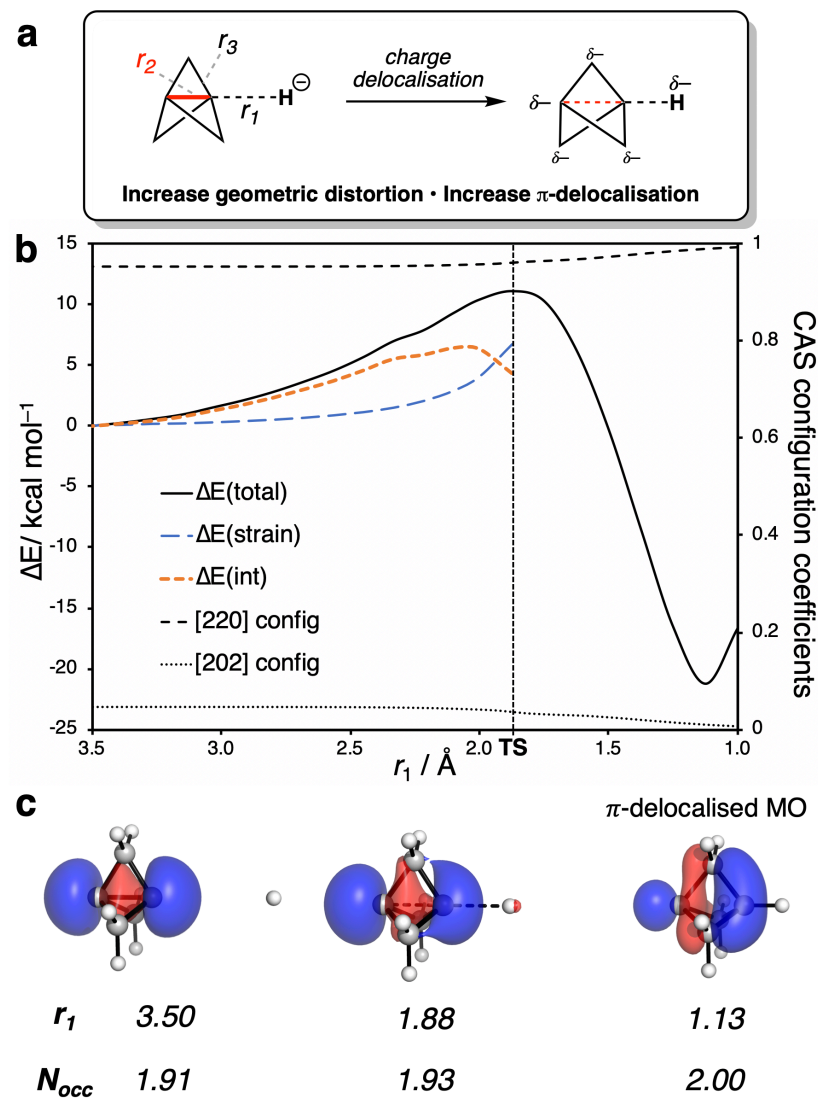

Figure 6. a. Addition of an anion $\left(\mathrm{H}^{-}\right)$to TCP (1) forces an expansion of $r_{2}$. b. Total energy (solid line), Activation Strain Analysis (ASA, blue and orange dashed lines) and configuration coefficients (dashed black lines) as a function of the reaction coordinate $\left(r_{1} / \AA\right)$, calculated for the addition of hydride to TCP (1) at $\operatorname{SMD}\left(\mathrm{Et}_{2} \mathrm{O}\right)$-DLPNO-CCSD(T)/ma-def2-QZVPP//CASSCF $(4,3) /$ ma-def2-QZVPP. c. Active MO showing the distortion from $\sigma$ to $\pi$ bonding along the reaction coordinate (isovalue $=0.05$ ). $N_{o c c}=\mathrm{MO}$ occupation number from CASSCF(4,3)/ma-def2-QZVPP calculation.

In TCP itself, static correlation (HOMO-LUMO mixing) acted to remove charge density from the center of the cage onto each apex carbon atom to relieve electronic repulsion. However, by distorting the $\mathrm{C}_{1}-\mathrm{C}_{3}$ bonding orbital to form a doubly-occupied $\pi$-delocalized MO (Fig. 6c, right), electron density was further forced away from the center of the cage, resulting in a loss of multireference character (Fig. 6b, black dashed/dotted lines).

From these analyses, addition of anions to TCP can be rationalized as follows: electrostatic repulsion between the approaching anion and TCP dominates at long- and medium-range. This 
repulsion leads to the formation of a doubly-occupied $\pi$-delocalized $\mathrm{MO}$, where the build-up of charge is spread over the cage. The cage then distorts to maximize this delocalization, and it is this distortion that is also partially responsible for the barrier to addition. This new paradigm is consistent with the reaction conditions required for the turbo-Hauser amide addition (vide supra).

\subsection{The reactivity of [1.1.1]propellane with radicals.}

We next turned our attention to the reactivity of TCP with radicals, given the prominence of this pathway in the synthesis of highly-functionalized BCPs by Wiberg, ${ }^{38}$ Michl, ${ }^{66}$ Uchiyama, ${ }^{29}$ Anderson $^{30,31}$ and Bräse. ${ }^{32}$ Such reactions proceed via the generation of a radical species that adds across the $\mathrm{C}_{1}-\mathrm{C}_{3}$ bond of $\mathbf{1}$, giving a bicyclo[1.1.1]pentyl radical that is subsequently trapped via either atom-transfer or addition to a radical trap (such as an azodicarboxylate or a further molecule of TCP). Alkoxycarbonyl, alkyl and aryl radical additions have previously been studied using DFT (B3LYP, M06-2X, $\omega$ B97X-D, B2PLYP) by Uchiyama ${ }^{29}$ and Anderson, ${ }^{30,31}$ with a focus on the fate of the bicyclo[1.1.1]pentyl radical adduct for further functionalization. In light of the importance of $\pi$-delocalization in the reactivity of TCP with anions (vide supra), we sought to further probe the initial radical addition reaction.

Cleavage of the $\mathrm{C}_{1}-\mathrm{C}_{3}$ bond in TCP by a radical species requires access to an open-shell doublet or quartet electronic configuration. TCP itself is not diradicaloid in nature, as confirmed by Salvador using CASSCF ${ }^{67}$ however, perturbation of the electronic structure of $\mathbf{1}$ by an approaching radical could act to enforce a 'more diradical' configuration. To investigate this, we employed CASSCF using the $(3,3)$ active space described in the Computational Methods (Fig. 3c). Upon the approach of the radical an increase in the doublet [111] electronic configuration (Fig. 7b, solid red line) was observed shortly before the transition state. This indeed is indicative of the formation of a more diradical structure, where electron density is displaced into an antibonding MO (see Computational Methods for a discussion of the active space). This effect came at the expense of the major [210] configuration (black dashed line) corresponding to the doubly-occupied new bonding MO. 


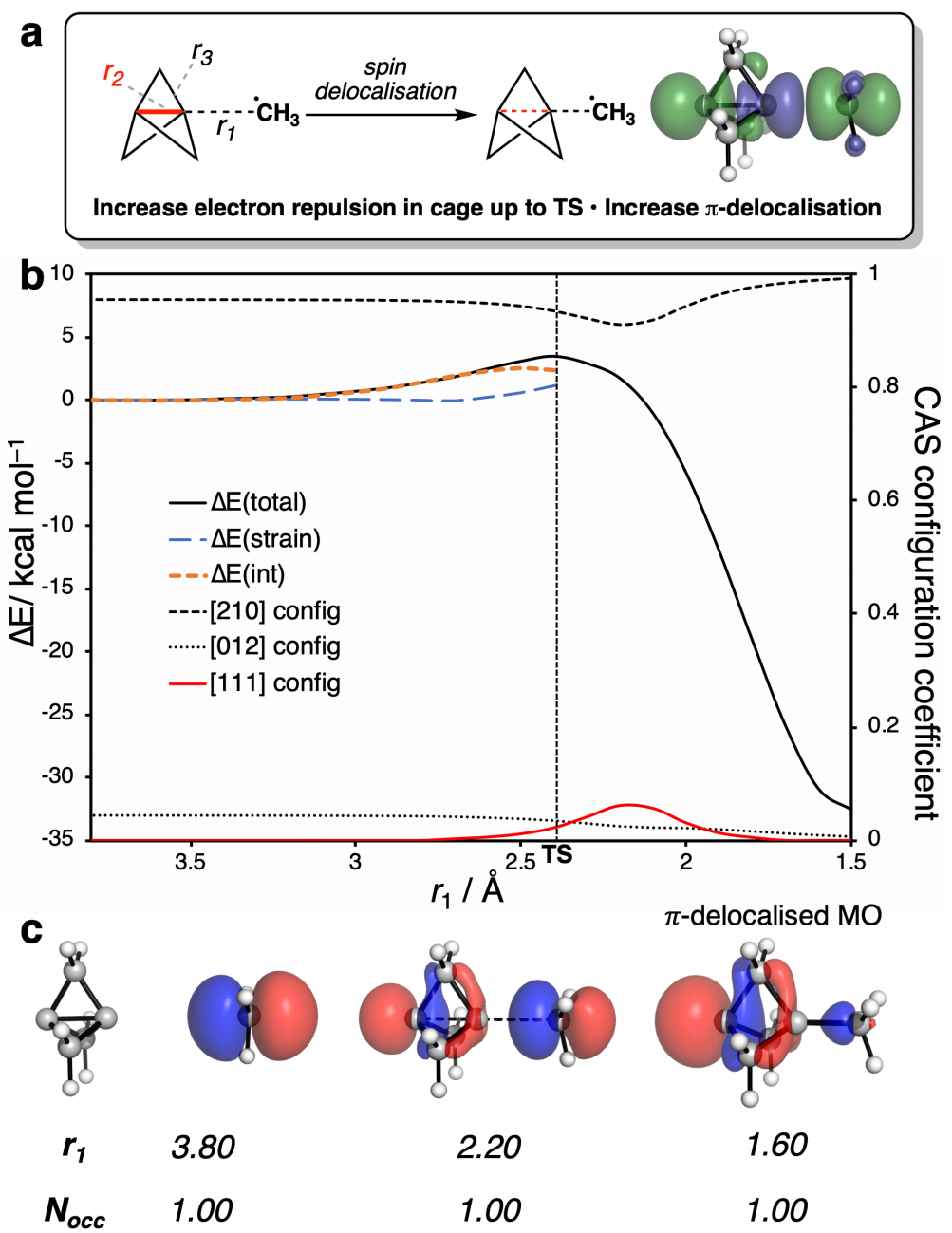

Figure 7. a. Addition of $\bullet \mathrm{CH}_{3}$ to TCP (1) increases electronic repulsion inside the cage, but is stabilized by $\pi$-delocalization. b. Activation Strain Analysis (ASA): total (black solid line), strain (blue dashed line) and interaction(orange dashed lines) energies; configuration coefficients (dashed black lines) as a function of the reaction coordinate $\left(r_{1} / \AA\right)$, calculated at $\operatorname{SMD}\left(\mathrm{Et}_{2} \mathrm{O}\right)$-DLPNO-CCSD(T)/def2QZVPP//CASSCF(3,3)/def2-QZVPP. c. Active molecular orbital (isovalue $=0.05$ ) as a function of $r_{l}(\AA)$, showing the development of $\pi$-character in the MO. $N_{o c c}=\mathrm{MO}$ occupation number from $\operatorname{CASSCF}(3,3) /$ def2-QZVPP calculation.

As for anionic addition, Activation Strain Analysis revealed that the barrier to addition was dominated by electronic effects ( $\Delta \mathrm{E}$ (int), Fig. $\mathbf{7 b}$, blue dashed line), rather than the increase in strain $(\Delta \mathrm{E}$ (strain), orange dashed line), although the effect was smaller here. We propose this electronic barrier occurs for two reasons: firstly, that the initial approach of the radical species forces an increase in electron density inside the cage before any electronic reorganization can occur, and secondly that electron density must be lost from the forming bond to allow for the interaction of the electron spins. To counter these effects, development of $\pi$-delocalization in the singly-occupied 
MO (Fig. 7c) acts to spread the additional electron density over the vertex carbon atoms, an effect that can be seen by the calculation of the spin density at the transition state depicted in Fig. 7a.

To probe the consequences of this reactivity in an experimental system, the diiodination of TCP was chosen as a model reaction, not least given its utility as a method for the quantification of the concentration of solutions of $\mathbf{1}$. We studied this process using geometries optimized with the B2PLYP double-hybrid density functional with empirical dispersion (D3BJ) and the SMD solvation model (see Computational Methods). This reaction is commonly assumed to occur via addition of I• to TCP to form an iodobicyclo[1.1.1]pentyl radical intermediate (D, Fig. 8a), which is then trapped by $\mathrm{I}_{2}$, affording 1,3-diiodobicyclo[1.1.1]pentane (E) and regenerating $\mathrm{I} \bullet$ as a chain carrier.

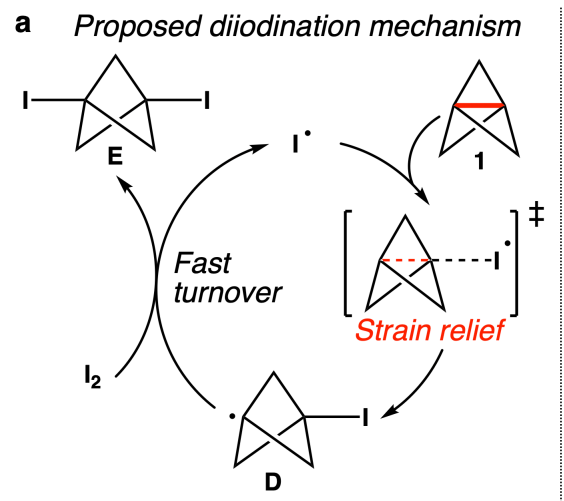

b Revised mechanism

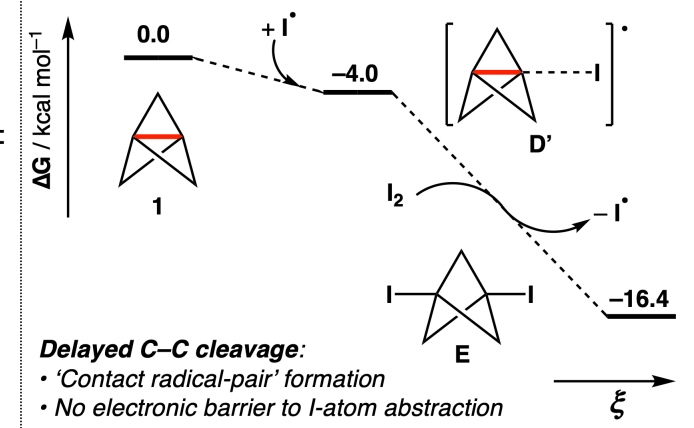

c
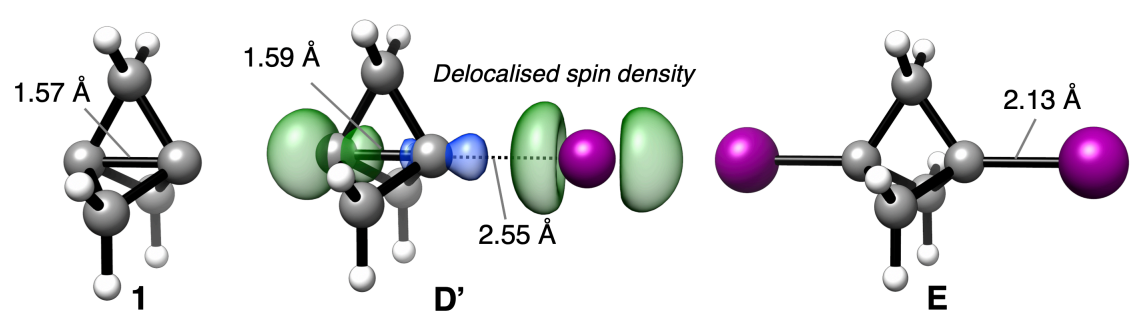

Figure 8. a. Proposed mechanism for the diiodination of $\mathbf{1}$. b. Free energy profile for the diiodination of $\mathbf{1}$, calculated at the $\mathrm{SMD}\left(\mathrm{Et}_{2} \mathrm{O}\right)$-DLPNO-CCSD(T)/def2-QZVPP//SMD(Et $\left.{ }_{2} \mathrm{O}\right)-\mathrm{B} 2 \mathrm{PLYP}-\mathrm{D} 3 \mathrm{BJ} / \mathrm{def}_{2}-\mathrm{TZVP}$ level of theory. c. Geometries of intermediate species, calculated at the $\operatorname{SMD}\left(\mathrm{Et}_{2} \mathrm{O}\right)-\mathrm{B} 2 \mathrm{PLYP}-\mathrm{D} 3 \mathrm{BJ} / \mathrm{def} 2-$ TZVP level of theory. Spin density is plotted at an isovalue of 0.01 a.u.

However, on studying this reaction computationally, the initial addition of $\mathrm{I} \bullet \operatorname{did}$ not proceed to the expected carbon-centered radical (Fig. 8b); instead, weak association of the iodine radical to 
TCP (1) was favored over of full $\mathrm{C}_{1}-\mathrm{C}_{3}$ bond cleavage, where only a slight increase in this length was observed in D' compared with TCP $\left(\Delta r_{2}=0.02 \AA\right)$. This barrierless exergonic complexation $\left(\Delta \mathrm{G}=-4.0 \mathrm{kcal} \mathrm{mol}^{-1}\right)$ was followed by atom-transfer with $\mathrm{I}_{2}$, which was also found to be electronically barrierless. The observation of significant spin density delocalization in the radical adduct D' suggested that while the geometry of 1 was not significantly distorted, $\mathrm{C}_{1}-\mathrm{C}_{3}$ bond-weakening occurred to accommodate the unpaired electron, accounting for the ease of attack of iodine atom abstraction from $\mathrm{I}_{2}$ through the distal carbon atom.

Extending this analysis to the other halogen radicals $(\mathrm{X}=\mathrm{F} \bullet, \mathrm{Cl} \bullet, \mathrm{Br} \bullet)$ revealed that as the strength of the $\mathrm{C}-\mathrm{X}$ bond increased, the addition reaction became more facile (Fig. 9a). In contrast to the iodine radical, these radicals were capable of fully cleaving the $C_{1}-C_{3}$ bond directly. A similar but more pronounced effect was observed with chalcogen hydride radicals (Fig. 9b), of which related thio- and selenoradical addition reactions have been extensively studied by Bräse. ${ }^{32}$ The complexation effect observed with $\mathrm{I} \bullet$ was magnified in the addition of $\bullet \mathrm{TeH}$ (red line), where the difference in the equilibrium $\mathrm{C}$-Te length between the radical and saturated bicyclo[1.1.1]pentane $\left(r-r_{e}=0.63 \AA\right)$ is even greater than that with iodine $\left(r-r_{e}=0.42 \AA\right.$ for I), suggesting an increase in electronegativity also plays a role in forming a stronger complex with $\mathbf{1}$. The propensity for bond cleavage versus complexation is purely thermodynamic in nature; the formation of a strong $\mathrm{C}-\mathrm{X}$ bond will overcome the $\mathrm{C} 1-\mathrm{C} 3$ bond of 1 , but a weak $\mathrm{C}-\mathrm{X}$ bond will not.

These results introduce the idea that TCP may, in fact, undergo reversible radical reactions, again challenging the concept of rapid strain relief. Rather, the ability of the cage to form a $\pi$-delocalized structure upon the addition of an electron drives the reaction. This raised the question of the consequence on reactivity when electrons were instead removed from the cage. 

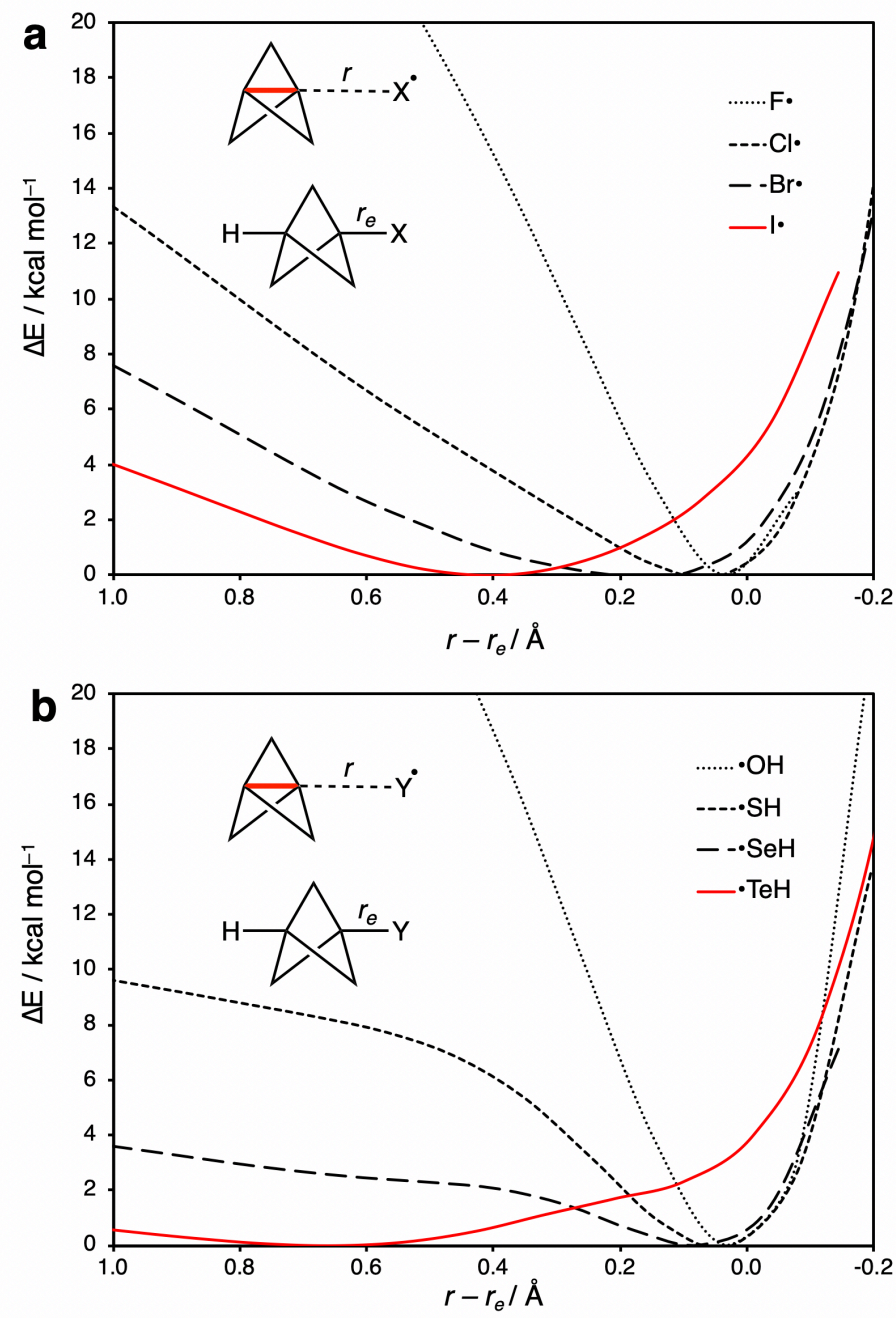

Figure 9. a. Potential energy surface for the addition of halide radicals $(\mathrm{X}=\mathrm{F} \bullet, \mathrm{Cl} \bullet \mathrm{Br} \bullet$ and $\mathrm{I} \bullet)$ to $\mathbf{1}$. b. Potential energy surface for the addition of chalcogen radicals $(\mathrm{Y}=\bullet \mathrm{OH}, \bullet \mathrm{SH}, \bullet \mathrm{SeH}$ and $\bullet \mathrm{TeH})$ to $\mathbf{1} . r-r_{e}$ denotes the difference between the forming $\mathrm{C}-\mathrm{X} / \mathrm{C}-\mathrm{Y}$ bond length and the equilibrium $\mathrm{C}-\mathrm{X} / \mathrm{C}-\mathrm{Y}$ bond length in the parent substituted bicyclo[1.1.1]pentane, calculated at the $\operatorname{SMD}\left(\mathrm{Et}_{2} \mathrm{O}\right)-\mathrm{B} 2 \mathrm{PLYP}-\mathrm{D} 3 \mathrm{BJ} / \mathrm{def} 2-$ TZVP level of theory.

\subsection{The reactivity of [1.1.1]propellane with cations.}

The cationic reactivity of $\mathbf{1}$ is an underdeveloped field. Despite the reported observation of rapid protonation of 1 with acetic acid, ${ }^{68}$ no further development of other cation-promoted reactions has been published. The fate of the resultant bicyclo[1.1.1]pentyl cations has been the focus of investigations by Wiberg and Anderson. ${ }^{31,64}$ These cationic adducts are known to rapidly fragment to bicyclo[1.1.0]butyl-1-carbinyl cations (F, Fig. 10a), which is suggestive of a significant change in 
the electronics of the cage scaffold. We studied this system using geometries optimized at the DSD-BLYP level of theory (see Computational Methods).

Activation Strain Analysis of this system revealed a contrasting picture to that seen with anionic and radical additions (vide supra). Instead of charge being forced into the cage causing an increase in $\Delta \mathrm{E}$ (int), now the donation of electron density to the approaching cation gave the opposite effect. Charge was lost from the cage, and no transition state was observed as the geometric distortion along the reaction coordinate $[\Delta \mathrm{E}$ (strain), Fig. 10b, dashed blue line] was minimal in comparison with the dominant stabilizing electronic interactions [ $\Delta \mathrm{E}(\mathrm{int})$, dashed orange line].

Examining the composition of the $(2,3)$ active space (Fig. 3b) calculated at the CASSCF level of theory revealed that the coefficient of the antibonding state [020] decreased upon approach of the cation (Fig. 10b). As a result, the $\mathrm{C}_{1}-\mathrm{C}_{3}$ bond decreased in length instead of undergoing cleavage, which had a consequence on cage integrity. An analogous phenomenon has been observed by Jemmis in calculations of halogen-bonded complexes of $\mathbf{1}$, where the removal of electron density from the cage did indeed strengthen the $\mathrm{C}_{1}-\mathrm{C}_{3}$ bond (B3LYP-D3BJ/QZ4P). ${ }^{10}$

This bond-shortening resulted in lateral compression of the cage, and an increase in the $\mathrm{C}_{1}-\mathrm{C}_{2}$, $\mathrm{C}_{1}-\mathrm{C}_{4}$ and $\mathrm{C}_{1}-\mathrm{C}_{5}\left(r_{3}\right)$ bond lengths (Fig. 10a). In effect, the electron density responsible for charge transfer to the cation derived from these 'wing' bonds, resulting in the formation of a highly distorted cage that is primed to fragment along $r_{3}$. Such behavior is consistent with the experimentally-observed rapid fragmentation of TCP to the bicyclo[1.1.0]butyl-1-carbinyl cation under acidic conditions. ${ }^{68}$ Once again, $\pi$-delocalization proved crucial for the reactivity of $\mathbf{1}$; however, in contrast to the anionic and radical reactivity profiles, here it is the loss of $\pi$-delocalization that mediated reactivity. This reactivity profile, while often associated with decomposition, may in fact provide the basis for a useful mode of reactivity with TCP. 

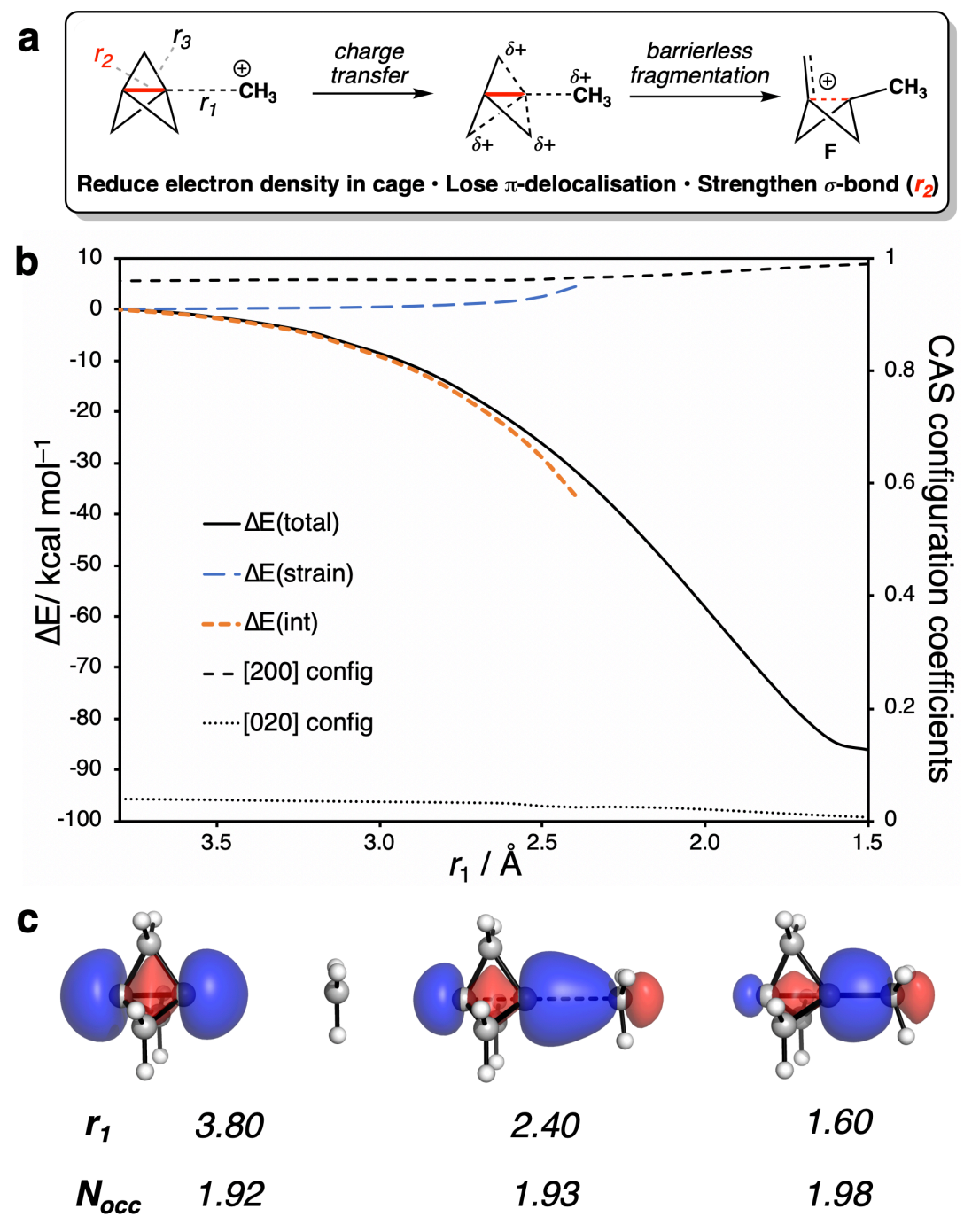

Figure 10. a. Addition of a cation to 1 reduces electronic repulsion inside the cage. Fragmentation of the $\mathrm{C}_{1}-\mathrm{C}_{2}$ bond is barrierless due to a loss of transannular $\pi$-bonding. b. Total energy (solid line), Activation Strain Analysis (ASA, blue and orange dashed lines) and configuration coefficients (dashed black lines) as a function of the reaction coordinate $\left(r_{I} / \AA\right)$, calculated for the addition of $\mathrm{CH}_{3}{ }^{+}$to TCP (1) at $\operatorname{SMD}\left(\mathrm{Et}_{2} \mathrm{O}\right)$-DLPNO-CCSD(T)/def2-QZVPP//CASSCF(2,3)/def2QZVPP. c. Active molecular orbital (isovalue $=0.05$ ) as a function of $r_{l}(\AA)$, showing a strengthening of the $\mathrm{C}_{1}-\mathrm{C}_{3}$ bond as $r_{3}$ elongates. $N_{o c c}=\mathrm{MO}$ occupation number from $\operatorname{CASSCF}(2,3) / \mathrm{def} 2-$ QZVPP calculation. 


\subsection{Unified model of reactivity and implications for synthetic applications of [1.1.1]propellane.}

As discussed in the Introduction, TCP (1) is omniphilic, in that the $\mathrm{C}_{1}-\mathrm{C}_{3}$ bridgehead bond can undergo various reactions with anions, radicals and cations to afford BCPs and exo-methylenecyclobutanes. In this work, we provide a unified picture to understand the origin of such promiscuity and its implications on the reactivity of TCP. Employing state-of-the-art computational approaches and energy partition schemes, we have identified suitable computational methodologies to explore the reactivity of TCP. Quantitative analysis regarding steric and electronic effects on the reaction of TCP with anions, radicals and cations is also provided. Our main findings can be summarized as follows:

1. The ground state structure of TCP is stabilized by the mixing of $\sigma$-bonding and antibonding electronic configurations. Electronic repulsion in the cage is reduced via delocalization into a $\pi$-bonding MO (Fig. 11a)

2. Intuitively, electronic repulsion should hinder anionic reactivity. However, significant electron delocalization in the forming adduct allows facile addition of anions to TCP with minimal structural distortion. Electronic reorganization takes place through the population of a doubly-occupied $\pi$-bonding MO in the addition process (Fig. 11a). These findings are supported by Activation Strain Analysis that predicts an unprecedented large and positive value for $\Delta \mathrm{E}$ (int) in these cases, with relatively little reorganization of the geometry of the cage (Fig. 11b).

3. While electronic repulsion is less significant for the radical process (Fig. 11b), there is an energetic cost associated with the formation of a species with diradical character: partial population of a $\sigma$-antibonding is required to allow spin-pairing to occur during bond formation. Through analysis of CAS orbitals and spin density distribution, we observed the stabilization of the adduct via occupation of a singly-occupied $\pi$-bonding MO (Fig. 11a).

4. Cationic reactivity is dominated by the loss of electron density from the cage. Despite the small cost of geometric reorganization, the addition process is barrierless as electronic repulsion in the cage is lost (Fig. 11b). The bridgehead $\mathrm{C}_{1}-\mathrm{C}_{3}$ bond is strengthened by a decrease in the population of its antibonding orbital (Fig. 11a). The now charge-deficient 
cage is stabilized by the removal of electron density from the $\mathrm{C}_{1}-\mathrm{C}_{2}, \mathrm{C}_{1}-\mathrm{C}_{4}$ and $\mathrm{C}_{1}-\mathrm{C}_{5}$ bonds (red dashed lines), resulting in fragmentation.

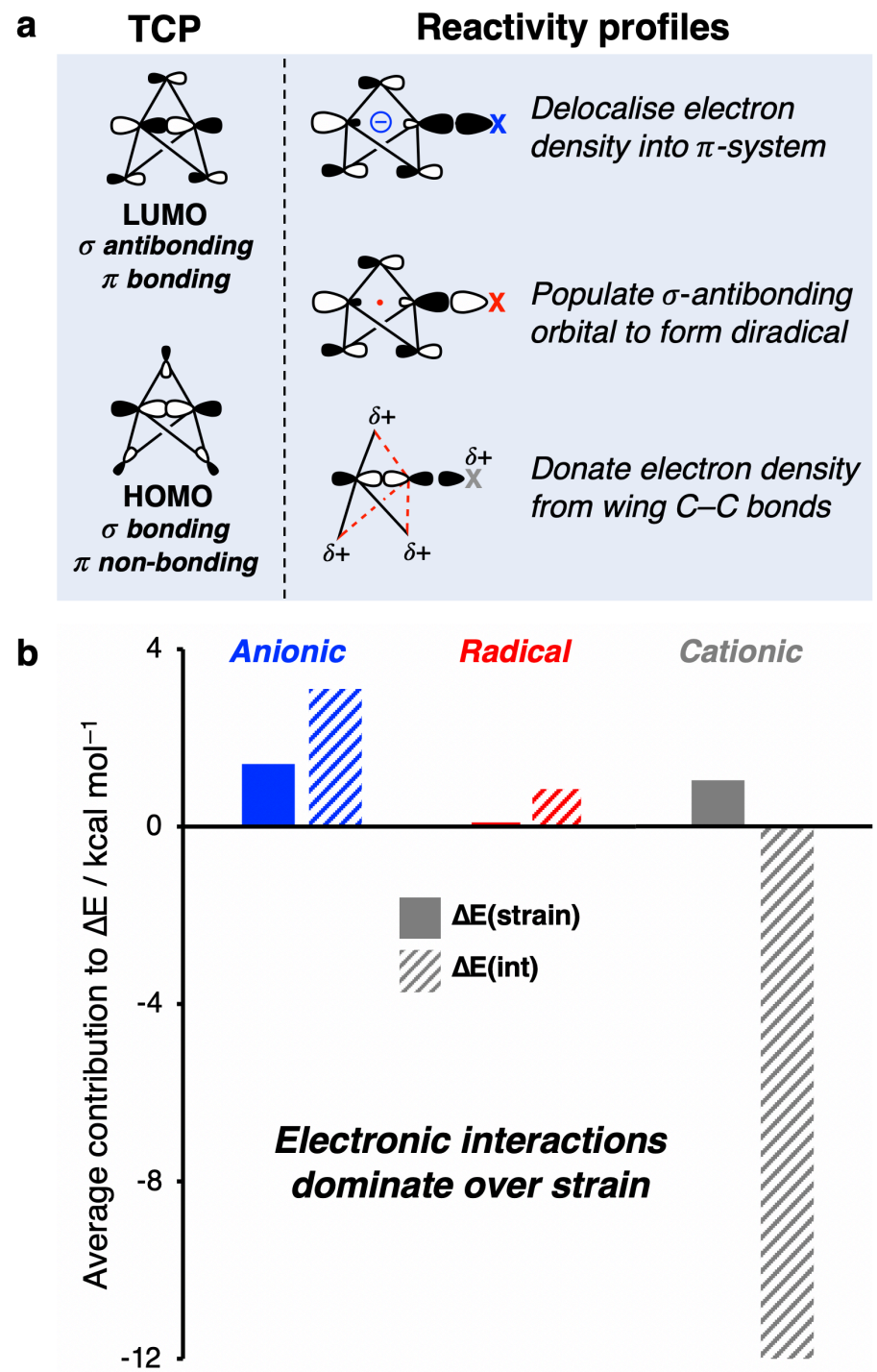

Figure 11. a. Summary of the omniphilic reactivity profile of TCP (1). b. Average contribution of $\Delta \mathrm{E}$ (strain) [solid bars] and $\Delta \mathrm{E}$ (int) [striped bars] to the anionic, radical and cationic activation profiles $[\Delta \mathrm{E}]$.

These analyses define the omniphilic reactivity profile of TCP, giving a generalizable interpretation of the reactivity of this promiscuous, and now predictable, molecule. We anticipate that these observations will be of use to inspire the development of new methodologies with TCP. For example, by rationalizing the electrophilic reactivity profile of TCP, we hope to promote the design of novel methodologies that capitalize on the unique structural distortion of the cage upon a loss 
of electronic repulsion. In addition, the formation of radical-pair complexes with heavy atoms provides a platform for reversible reactivity with TCP. We also predict that substitution of the apex carbon atoms, either with heteroatoms or elaborated carbon-based fragments, will alter the behavior and electronic structure of TCP both in the ground state and upon reaction. The consequence of substitution on strain in [1.1.1]propellanes has been investigated computationally by Pittman; ${ }^{69}$ Szeimes et al. have also experimentally investigated differing thermal rearrangements of substituted propellanes. ${ }^{70} \mathrm{We}$ expect a similarly diverse range of bimolecular reactions with substituted TCPs, where cage substitution is likely to substantially modulate reactivity with charged species. With these points in mind, the development of new analogues and reactions of TCP will provide a source of functionality-rich building blocks that may find further use in both pharmaceutical and materials settings.

\section{Conclusions}

In depth analysis of the structure and reactivity of [1.1.1]propellane (1) reveals that its reactions with anions, radicals and cations are not dictated by relief of strain - barriers to addition are dominated by the redistribution of electron density into and out of $\pi$-delocalized molecular orbitals. These unprecedented results highlight the benefits of the theoretical approach underpinning this work: looking beyond DFT, we have shown that analysis of multi-reference calculations provides unparalleled insight into the physical principles governing reactivity.

A computational survey showed that both coupled-cluster [DLPNO-CCSD(T)] methods and double-hybrid density functionals [B2PLYP-D3BJ, B2GP-PLYP-D3BJ, DSD-BLYP] described the important static correlation effects needed to accurately model the electronic structure and reactivity of [1.1.1]propellane. While a cancellation of errors was seen to favor the performance of single-hybrid DFT (M06-2X, $\omega$ B97X-D3) in the reactions studied here, caution should be exercised when applying these methods onto the calculation of even very similar systems; systemdependent overlocalization of electron density inside the cage resulted in incorrect behavior of the bridgehead $\mathrm{C}-\mathrm{C}$ bond, despite good agreement near the transition state in each case. 
This work is the first dedicated theoretical investigation into the origins of the reactivity of [1.1.1]propellane, where we anticipate that this new understanding will open up possibilities for the development of novel reactivity of [1.1.1]propellane and related systems. Ultimately, we hope that the concepts presented here will provide synthetic chemists with the understanding to develop new methods to rapidly access structures that are fast moving into the mainstream of medicinal and materials chemistry.

\section{Supporting Information}

The Supporting Information includes: Computational methods and data including benchmarking studies, Figures S1-S22, Tables S1-S48, Cartesian coordinates (PDF); Cartesian coordinates (ZIP)

\section{AUTHOR INFORMATION}

Corresponding Authors:

*fernanda.duartegonzalez@chem.ox.ac.uk;

*edward.anderson@chem.ox.ac.uk

\section{Author Contributions}

The work was conceived by A.J.S., E.A.A. and F.D. Calculations and data analysis were carried out by A.J.S. The manuscript was written through contributions of all authors. All authors have given approval to the final version of the manuscript.

\section{Funding Sources}

A.J.S. thanks the EPSRC Centre for Doctoral Training in Synthesis for Biology and Medicine for a studentship (EP/L015838/1), generously supported by AstraZeneca, Diamond Light Source, Defence Science and Technology Laboratory, Evotec, GlaxoSmithKline, Janssen, Novartis, Pfizer, Syngenta, Takeda, UCB and Vertex. A.J.S. also thanks the Oxford-Radcliffe Scholarship for a student-ship. A.J.S. and F.D. thank the EPSRC Tier-2 National HPC Facility Service (http://www.cirrus.ac.uk), and the EPSRC Centre for Doctoral Training for Theory and Modelling in Chemical Sciences (EP/L015722/1) for providing access to the Dirac cluster at Oxford. E.A.A. thanks for EPSRC for support (EP/S013172/1). 


\section{ACKNOWLEDGMENT}

We thank Prof. Igor V. Alabugin, Prof. John E. McGrady, Dr Alex J. W. Thom, Dr Alexander B.

Dürr, Mr Harry W. T. Morgan and Mr Tom A. Young for insightful discussions.

\section{REFERENCES}

(1) Wiberg, K. B.; Walker, F. H. J. Am. Chem. Soc. 1982, 104 (19), 5239.

(2) Honegger, E.; Huber, H.; Heilbronner, E.; Dailey, W. P.; Wiberg, K. B. J. Am. Chem. Soc. 1985, 107 (24), 7172.

(3) Jackson, J. E.; Allen, L. C. J. Am. Chem. Soc. 1984, 106 (3), 591.

(4) Wiberg, K. B.; Walker, F. H.; Pratt, W. E.; Michl, J. J. Am. Chem. Soc. 1983, 105 (11), 3638.

(5) Walker, F. H.; Wiberg, K. B.; Michl, J. J. Am. Chem. Soc. 1982, 104 (7), 2056.

(6) Kar, T.; Jug, K. Chem. Phys. Lett. 1996, 256, 201.

(7) Bremer, M.; Untenecker, H.; Gunchenko, P. A.; Fokin, A. A.; Schreiner, P. R. J. Org. Chem. 2015, 80 (12), 6520.

(8) Messerschmidt, M.; Scheins, S.; Grubert, L.; Pätzel, M.; Szeimies, G.; Paulmann, C.; Luger, P. Angew. Chem. Int. Ed. 2005, 44 (25), 3925.

(9) Laplaza, R.; Contreras-Garcia, J.; Fuster, F.; Volatron, F. 1.

(10) Joy, J.; Akhil, E.; Jemmis, E. D. Phys. Chem. Chem. Phys. 2018, 20 (40), 25792.

(11) Polo, V.; Andres, J.; Silvi, B. J. Comput. Chem. 2007, 28, 857.

(12) Yang, Y. J. Phys. Chem. A 2012, 116 (41), 10150.

(13) Wu, W.; Gu, J.; Song, J.; Shaik, S.; Hiberty, P. C. Angew. Chem. Int. Ed. 2009, 48 (8), 1407.

(14) Mu, B.; Bally, T.; Pappas, R.; Williams, F. J. Am. Chem. Soc. 2010, 132, 14649.

(15) Levin, M. D.; Kaszynski, P.; Michl, J. Chem. Rev. 2000, 100 (1), 169.

(16) Dilmaç, A. M.; Spuling, E.; de Meijere, A.; Bräse, S. Angew. Chem. Int. Ed. 2017, 56 (21), 5684.

(17) Stepan, A. F.; Subramanyam, C.; Efremov, I. V.; Dutra, J. K.; O’Sullivan, T. J.; Dirico, K. J.; McDonald, W. S.; Won, A.; Dorff, P. H.; Nolan, C. E.; Becker, S. L.; Pustilnik, L. R.; Riddell, D. R.; Kauffman, G. W.; Kormos, B. L.; Zhang, L.; Lu, Y.; Capetta, S. H.; Green, M. E.; Karki, K.; Sibley, E.; Atchison, K. P.; Hallgren, A. J.; Oborski, C. E.; Robshaw, A. E.; Sneed, B.; O’Donnell, C. J. J. Med. Chem. 2012, 55 (7), 3414.

(18) Measom, N. D.; Down, K. D.; Hirst, D. J.; Jamieson, C.; Manas, E. S.; Patel, V. K.; Somers, D. O. ACS Med. Chem. Lett. 2017, 8 (1), 43.

(19) Auberson, Y. P.; Brocklehurst, C.; Furegati, M.; Fessard, T. C.; Koch, G.; Decker, A.; La Vecchia, L.; Briard, E. ChemMedChem 2017, 12 (8), 590.

(20) Goh, Y. L.; Cui, Y. T.; Pendharkar, V.; Adsool, V. A. ACS Med. Chem. Lett. 2017, 8(5), 516.

(21) Nicolaou, K. C.; Vourloumis, D.; Totokotsopoulos, S.; Papakyriakou, A.; Karsunky, H.; Fernando, H.; Gavrilyuk, J.; Webb, D.; Stepan, A. F. ChemMedChem 2016, 11 (1), 31.

(22) Wang, J.; Lundberg, H.; Asai, S.; Martín-Acosta, P.; Chen, J. S.; Brown, S.; Farrell, W.; Dushin, R. G.; O’Donnell, C. J.; Ratnayake, A. S.; Richardson, P.; Liu, Z.; Qin, T.; Blackmond, D. G.; Baran, P. S. Proc. Natl. Acad. Sci. 2018, 115 (28), E6404.

(23) Makarov, I. S.; Brocklehurst, C. E.; Karaghiosoff, K.; Koch, G.; Knochel, P. Angew. Chem. Int. Ed. 2017, 56 (41), 12774.

(24) Westphal, M. V.; Wolfstädter, B. T.; Plancher, J. M.; Gatfield, J.; Carreira, E. M. ChemMedChem 
2015, $10(3), 461$.

(25) Itzhaki, L.; Altus, E.; Basch, H.; Hoz, S. Angew. Chem. Int. Ed. 2005, 44 (45), 7432.

(26) Schwab, P. F. H.; Noll, B. C.; Michl, J. J. Org. Chem. 2002, 67 (16), 5476.

(27) De Meijere, A.; Messner, M. Mol. Cryst. Liq. Cryst. 1994, 257 (1), 161.

(28) De Meijere, A.; Ligang, Z.; Belov, V. N.; Bossi, M.; Noltemeyer, M.; Hell, S. W. Chem. Eur. J. 2007, 13 (9), 2503.

(29) Kanazawa, J.; Maeda, K.; Uchiyama, M. J. Am. Chem. Soc. 2017, 139 (49), 17791.

(30) Caputo, D. F. J.; Arroniz, C.; Dürr, A.; Mousseau, J.; Stepan, A.; Mansfield, S.; Anderson, E. A. Chem. Sci. 2018, 9, 5295.

(31) Nugent, J.; Arroniz, C.; Shire, B. R.; Sterling, A. J.; Pickford, H. D.; Wong, L. J.; Mansfield, S. J.; Caputo, D. F. J.; Owen, B.; Mousseau, J. J.; Duarte, F.; Anderson, E. A. Prepr. 2019, 1.

(32) Bär, R. M.; Kirschner, S.; Nieger, M.; Bräse, S. Chem. Eur. J. 2018, 24 (6), 1373.

(33) Gianatassio, R.; Lopchuk, J. M.; Wang, J.; Pan, C.; Malins, L. R.; Prieto, L.; Brandt, T. A.; Collins, M. R.; Gallego, G. M.; Sach, N. W.; Spangler, J. E.; Zhu, H.; Zhu, J.; Baran, P. S. Science 2016, 351 (6270), 241.

(34) Lopchuk, J. M.; Fjelbye, K.; Kawamata, Y.; Malins, L. R.; Pan, C. M.; Gianatassio, R.; Wang, J.; Prieto, L.; Bradow, J.; Brandt, T. A.; Collins, M. R.; Elleraas, J.; Ewanicki, J.; Farrell, W.; Fadeyi, O. O.; Gallego, G. M.; Mousseau, J. J.; Oliver, R.; Sach, N. W.; Smith, J. K.; Spangler, J. E.; Zhu, H.; Zhu, J.; Baran, P. S. J. Am. Chem. Soc. 2017, 139 (8), 3209.

(35) Shelp, R. A.; Walsh, P. J. Angew. Chem. Int. Ed. 2018, 57 (48), 15857.

(36) Trongsiriwat, N.; Pu, Y.; Nieves-Quinones, Y.; Shelp, R. A.; Kozlowski, M. C.; Walsh, P. J. Angew. Chem. Int. Ed. 2019, 1.

(37) Hedberg, L.; Hedberg, K. J. Am. Chem. Soc. 1985, 107 (25), 7257.

(38) Wiberg, K. B.; Waddell, S. T. J. Am. Chem. Soc. 1990, 112 (6), 2194.

(39) Wiberg, K. B. Angew. Chemie Int. Ed. English 1986, 25 (4), 312.

(40) Bickelhaupt, F. M.; Houk, K. N. Angew. Chem. Int. Ed. 2017, 56 (34), 10070.

(41) Becke, A. D. J. Chem. Phys. 1993, 98, 5648.

(42) Zhao, Y.; Truhlar, D. G. Theor. Chem. Acc. 2008, 120 (1-3), 215.

(43) Cohen, A. J.; Mori-Sánchez, P.; Yang, W. Chem. Rev. 2012, 112 (1), 289.

(44) Mardirossian, N.; Head-Gordon, M. Mol. Phys. 2017, 115 (19), 2315.

(45) Malmqvist, P.; Roos, B.; Andersson, K. J. Chem. Phys. 1992, 96, 1218.

(46) Angeli, C.; Cimiraglia, R.; Evangelisti, S.; Leininger, T.; Malrieu, J. P. J. Chem. Phys. 2001, 114 (23), 10252.

(47) Szalay, P. G.; Müller, T.; Gidofalvi, G.; Lischka, H.; Shepard, R. Chem. Rev. 2012, 112 (1), 108.

(48) Phung, Q. M.; Feldt, M.; Harvey, J. N.; Pierloot, K. J. Chem. Theory Comput. 2018, 14 (5), 2446.

(49) Van Zeist, W. J.; Bickelhaupt, F. M. Org. Biomol. Chem. 2010, 8 (14), 3118.

(50) Neese, F. Wiley Interdiscip. Rev. Comput. Mol. Sci. 2018, 8 (1), 4.

(51) Weigend, F.; Ahlrichs, R. Phys. Chem. Chem. Phys. 2005, 7 (18), 3297.

(52) Liakos, D. G.; Neese, F. J. Chem. Theory Comput. 2015, 11 (9), 4054.

(53) Grimme, S. J. Chem. Phys. 2006, 124, 034108.

(54) Grimme, S.; Antony, J.; Ehrlich, S.; Krieg, H. J. Chem. Phys. 2010, 132, 154104.

(55) Johnson, E. R.; Becke, A. D. J. Chem. Phys. 2005, 123, 024101.

(56) Karton, A.; Tarnopolsky, A.; Schatz, G. C.; Martin, J. M. L. J. Phys. Chem. A 2008, 112, 12868.

(57) Kozuch, S.; Gruzman, D.; Martin, J. M. L. J. Phys. Chem. C 2010, 114 (48), 20801. 
(58) Marenich, A. V; Cramer, C. J.; Truhlar, D. G. J. Phys. Chem. B. 2009, 113, 6378.

(59) Grimme, S. Chem. Eur. J. 2012, 18 (32), 9955.

(60) Seiler, P. Helv. Chim. Acta 1990, 73, 1574.

(61) Feller, D.; Davidson, E. R. J. Am. Chem. Soc. 1987, 109 (14), 4133.

(62) Becke, A. D. Phys. Rev. A 1988, 38 (6), 3098.

(63) Lee, C.; Yang, W.; Parr, R. G. Phys. Rev. B 1988, 37 (2), 785.

(64) Wiberg, K. B.; McMurdie, N. J. Am. Chem. Soc. 1994, 116 (26), 11990.

(65) Messner, M.; Kozhushkov, S. I.; De Meijere, A. Eur. J. Org. Chem. 2000, No. 7, 1137.

(66) Kaszynski, P.; Friedli, A. C.; Michl, J. J. Am. Chem. Soc. 1992, 114 (2), 601.

(67) Ramos-Cordoba, E.; Salvador, P. Phys. Chem. Chem. Phys. 2014, 16, 9565.

(68) Wiberg, K. B.; Dailey, W. P.; Walker, F. H.; Waddell, S. T.; Crocker, L. S.; Newton, M. J. Am. Chem. Soc. 1985, 107 (25), 7247.

(69) Xu, H.; Saebo, S.; Pittman, C. U. Mol. Phys. 2012, 110 (19-20), 2349.

(70) Belzner, J.; Szeimies, G. Tetrahedron Lett. 1986, 27 (48), 5839. 